\title{
Synthesis and Modification of Polypropylene by Radiation-induced Grafting
}

\author{
Feng Yuan, Junfu Wei (Corresponding author), En-qi Tang, Kong-yin Zhao \& Yang Xue \\ Tianjin Municipal Key Lab of Fiber Modification and Functional Fibers \\ Tianjin 300160, China \\ E-mail: jfwei@tjpu.edu.cn \\ School of Material Science and Chemical Engineering \\ Tianjin Polytechnic University \\ Tianjin 300160, China \\ E-mail: yuanfeng840106@hotmail.com
}

\begin{abstract}
Polypropylene fiber (PP) was radiation-induced graft polymerization of lauryl acrylate (LA) and butyl acrylate (BA) using a ${ }^{60} \mathrm{Co}$ gamma radiation source. Then the grafted polypropylene fiber was characterized by a Fourier transform infrared (FT-IR) spectrometer, Scanning Electron Microscopy (SEM), differential scanning calorimetry (DSC) and thermogravimetry (TG), which indicated that LA and BA have been grafted onto PP. The article focused on some factors that influenced on the graft degree, such as the cross-linking agent concentration, monomer concentration. The results showed that: the degree of grafting BA reached the maximum of $20.53 \%$, while the maximum graft degree of LA was $29.90 \%$.
\end{abstract}

Keywords: Polypropylene, Butyl acrylate, Lauryl acrylate, Radiation grafting, Fiber

\section{Introduction}

Polymers have generated considerable interest as biomaterials in medical science and biotechnology. With increasing demands for a biomaterial with better acceptability and functionality to the biosystem, stress has been focussed on the development of newer materials. One of the ways to develop such materials is to modify existing polymers and design them keeping in view their specific application areas. Sutures are used in surgical operations and require optimum physico-chemical characteristics. Polypropylene (PP) is one of the widely used biostable sutures due to its optimum tensile strength and low level of tissue reaction. PP fibers possess good hydrophobic and lipophilic properties with lower density than water, which can suspend in the surface of water and have a high adsorption capacity of organic solvents such as hydrocarbons, esters, ketones, halohydrocarbons and so on. Therefore, Modification or introduction of new characteristics to those based on synthetic polymers such as polypropylene, can be achieved by several methods. Grafting, whether initiated chemically or by ionizing radiations, constitutes one of the methods that is being applied successfully. The radiation-induced graft polymerization of monomers such as acrylates, methacrylic acid, acrylamide and acrylonitrile onto PP has been reported to introduce hydrogel characteristics in the polymer for biomedical applications (Bhuvanesh, 2006, PP. 161-167, Park, 2006, PP.46-54, Vahdata, 2007, PP.787-793). Chen Xiaoting (2005, PP.536-54 ) reported that using acrylates, methacrylates as monomers, butylene glycol diacrylate as crosslinking agent, BPO as initiator, the high oil absorption resins polyacrylate were synthesized by suspension polymerization. The oil absorption ability of the resin is more than $15(\mathrm{~g} / \mathrm{g})$ for toluene, $10(\mathrm{~g} / \mathrm{g})$ for gasoline and $9(\mathrm{~g} / \mathrm{g})$ for machine oil. Zhang Yudong (1999, PP. 79-82)reported that grafting of ethyl acrylate(EA) onto PP powder had been carried out by preirradiation method using gamma rays from $3.7 \times 1014 \mathrm{~Bq}{ }^{60} \mathrm{Co}$ source as initiator. Chen Jie (1999, PP.193-198) reported that the surface of polypropylene (PP) film was modified by preirradiation grafting of polyethylene glycol methacrylate (PEGMA) with three kind of different molecular weight. Dong Yuan (2006, PP. 569-572) et al have indicatedt that the matrix of non-woven polypropylene fabrics could be grafted with the monomer of acrylic acid by means of pre-irradiation induced solid phase graft copolymerization. Jung-Dae Cho et al (2006, PP. 1446-1461)reported that 1,6-Hexanediol diacrylate (HDDA) was grafted onto polypropylene (PP) substrates in the presence of benzophenone (BP) using UV radiation and the UV-radiation grafting polymerizations were characterized in detail using contact-angle measurements, Fourier transform infrared spectroscopy with attenuated total internal reflection, and scanning electron microscopy. In the present study, the graft polymerization of LA and BA onto PP monofilament is 
carried out by a radition-induced grafting method to develop sutures with different graft levels.

\section{Materials and methods}

\subsection{Materials and reagents}

Commercial polypropylene fabric was kindly provided by Shijiazhuang Cigarette Industries Co. LTD. LA and BA purchased from Tianjiao Chemical Co., LTD, Tianjin, were used without further treatment. Other chemicals were analytical grade.

\subsection{Grafting and modification procedure}

Strips of PP films were immersed in monomer, cross-linking agents in glass ampoules. The reactant mixtures in glass ampoules were dearated by bubbling nitrogen gas for $10 \mathrm{~min}$, sealed and then subjected to gamma ray irradiation from ${ }^{60} \mathrm{Co}$. The grafted films thus obtained were removed and washed thoroughly with distilled water and then soaked overnight in acetone to eliminate the residual monomer and homopolymer contained in the films. The films were then dried in an oven for $24 \mathrm{~h}$ at $50-60^{\circ} \mathrm{C}$ and then weighed. The degree of grafting $G$ was calculated as follows:

$$
G(\%)=\left[\left(W g-W_{0}\right) / W_{0}\right] \times 100 \%
$$

where, $\mathrm{W}_{0}$ and $\mathrm{W}_{\mathrm{g}}$ represent, the weights of the initial and grafted films, respectively.

\subsection{FTIR measurement}

A Vector-22 Fourier transform infrared (FTIR) spectrometer, which is a product of Bruker, Co. Ltd, Germany, was used for measuring and scanning the infrared absorption spectra at resolution of $4 \mathrm{~cm}^{-1}$. High signal-to-noise spectra were obtained by collection of hundred scans for each sample. The resultant digitized spectra were stored for further data processing.

\subsection{SEM analysis}

The phase morphological characteristics of the samples were observed by means of SEM (HITACHI, S-3500N) in the normal secondary electron imaging (SEI) mode and all the membrane samples were coated with gold-palladium and fixed by adhesive tape in the sample stage.

\section{$2.5 T G$ analysis}

All samples were sheared to pieces and sputter-coated with Au. A NETZSCH STA409PC (Germany) thermogravimetry (TG) analyzer was used for thermal stability determinations of all samples under nitrogen atmosphere at a heating rate of $10^{\circ} \mathrm{C} / \mathrm{min}$ from $0^{\circ} \mathrm{C}$ to $600^{\circ} \mathrm{C}$.

\subsection{DSC analysis}

The thermal properties of PP composites were measured using a differential scanning calorimetry (Perkin-Elmer DSC-7, USA). Samples of about $10 \mathrm{mg}$ were heated from 0 to $200^{\circ} \mathrm{C}$, and then cooled down to $60{ }^{\circ} \mathrm{C}$ at the heating rate of $10{ }^{\circ} \mathrm{C}$ /min under an atmosphere of dry nitrogen.

\section{Results and discussion}

\subsection{Infrared spectroscopy measurement}

The presence of grafting of LA and BA onto PP film was confirmed by FTIR analysis. The IR spectra of virginal PP and grafted polymers PP-g-LA and PP-g-BA are given in Fig. 1. The appearance of the new bands at about 2960 2915 and $2870 \sim 2840 \mathrm{~cm}^{-1}$ on the pp matrix may be caused by the out-of-plane stretching vibrations and the symmetric stretching vibrations of the $\mathrm{CH}_{3}, \mathrm{CH}_{2}$, respectively; The appearance of the new adsorption peaks at about 1455 and $1375 \mathrm{~cm}^{-1}$ on the PP matrix may be caused by the $\mathrm{CH}_{3}$ out-of-plane bending vibrations and the symmetric bending vibrations of $\mathrm{CH}_{2}$, respectively. Compared to the spectrum of the original fiber, a strong adsorption peak at $1730 \mathrm{~cm}^{-1}$ can be observed, which characterizes the carbonyl group and caused by the stretching vibrations of carbonyl group of fatty acid esters, indicating that carbonyl has been grafted onto PP. The new characteristic absorption peak at $1150 \mathrm{~cm}^{-1}$ was corresponded to the stretching vibrations of $\mathrm{C}-\mathrm{O}$ and adsorption peaks at $1150 \mathrm{~cm}^{-1} \sim 1180 \mathrm{~cm}^{-1}$ and $1240 \mathrm{~cm}^{-1} \sim 1270 \mathrm{~cm}^{-1}$ was caused by the stretching vibrations of $\mathrm{C}-\mathrm{C}-\mathrm{O}-\mathrm{C}$. In combination with the vibration peak of carbonyl, it indicated that ester existed in the PP-g-LA and PP-g-BA matrix. A weak adsorption peak at about $720 \mathrm{~cm}^{-1}$ in $\mathrm{c}$ was caused by the bending vibration of $\mathrm{C}-\mathrm{H}$ in $\left(\mathrm{CH}_{2}\right)_{\mathrm{n}}(\mathrm{n} \geq 4)$, which indicated that branch chains of LA had been grafted onto PP.

Grafted PP films seems quite complicated at the adsorption peak of $1000 \mathrm{~cm}^{-1} \sim 1500 \mathrm{~cm}^{-1}$, due to the introduction of quaternary carbon ions after grafted, effects of carbonyl groups on the bending vibrations of methyl and the adsorption peak of $\mathrm{C}-\mathrm{O}-\mathrm{C}$ at $1000 \mathrm{~cm}^{-1} \sim 1300 \mathrm{~cm}^{-1}$.

Taken together, we can speculate that both monomer LA and BA have been grafted onto the virginal PP fibers.

\subsection{Scanning electron microscopy (SEM) study}

The surfaces of the virginal PP and the grafted PP films were examined by SEM, and the results are shown in Figure 2. 
The morphology of grafted PP is different significantly from that of virginal PP substrate. The grafted surface shows a markedly bumpy texture, while the virginal PP surface is very planar, which indicated that monomer LA and BA have been grafted onto the virginal PP fibers.

\subsection{TG analysis of PP matrix fibers}

Weight loss of virginal PP fiber and grafted fibers were measured at the heating rate of $10{ }^{\circ} \mathrm{C} / \mathrm{min}$ under an atmosphere of dry nitrogen. According to the TGA curves of the original PP fiber and the grafted fiber PP-g-LA and PP-g-BA given in Figures 3 and 4, the grafted PP fibers showed an excellent thermal stability at a temperature up to $370{ }^{\circ} \mathrm{C}$. As seen from the figure of TG and DTG, both PP-g-BA and PP-g-LA grafted fibers undergo two processes of thermal decomposition, corresponding to the decompositions of crylic acid grafted chains and original PP fibers, respectively. Grafted chains experienced decomposition at a temperature from 371.6 to $425{ }^{\circ} \mathrm{C}$, with a peak at $400.9^{\circ} \mathrm{C}$; At a temperature from 455.6 to $649.3^{\circ} \mathrm{C}$, original PP fibers experienced decomposition.

\subsection{DSC analysis of PP matrix fibers}

The melting temperature $\mathrm{Tm}$ and the apparent enthalpies of melting, $\triangle \mathrm{Hf}(\mathrm{fiber})$, were obtained from the maximum and the area of the melting peak, respectively. Similarly, the crystallization temperature (Tf) and crystallization enthalpy, $\triangle \mathrm{Hf}(\mathrm{cys})$ were obtained from the cooling DSC diagrams. The apparent crystallinity, $\mathrm{Wc}(\%)$, of the PP and the grafted PP were calculated by the following equation:

$\mathrm{Wc}(\%)=\triangle \mathrm{Hf}(\mathrm{PP}) / \triangle \mathrm{Hf}(\mathrm{cys}) \times 100$

where $\triangle \mathrm{Hf}(\mathrm{cys})$ is the heat of fusion per gram of $100 \%$ crystalline PP, which was $209 \mathrm{~J} / \mathrm{g}$ (Yin, 2001). Because crystallization of the grafted PP occurs in the PP portion, the crystallinity of PP portion in the grafted copolymer, $\triangle \mathrm{Hf}(\mathrm{PP})$ can be expressed as the following:

$\triangle \mathrm{Hf}(\mathrm{PP})=\triangle \mathrm{Hf}($ fiber $) /(1-\alpha)$

where $\alpha$ is the weight fraction of grafts in the grafted copolymer.

The melting and crystallization behaviors of the samples are shown in Figure 5.The Tm, $\triangle \mathrm{Hf}(\mathrm{PP}), \triangle \mathrm{Hf}(\mathrm{fiber})$ and Wc(\%) obtained from DSC curves in Figure 5 are listed in Table 1. It is known from these data that enthalpies of melting of grafted PP fibers decreased ascribed to "dilution effects" of grafts and "destructive effects" of radiation reaction on crystal zone (Huang, 2004, PP. 71-74, Zhu, 2004). Grafting of LA and BA onto PP film reduces the heat of melting and increases the melting enthalpy of PP. Influence of Tm is ascribed to two aspects. 1, grafting reaction was processed in the crazing zone of PP or main PP chains of crystal deficiency zone, regularity of crystal zone of PP got worse after grafted, destroyed the inherent crystal zone, which lead to the reduction of Tm of grafted PP fibers; 2 , grafted PP polymers formed a new crystal which have a different Tm from virginal PP fibers. When the melting point of grafted chains is lower than virginal PP or grafted chains can't form crystal, melting point of grafted PP decreases compared to the virginal PP. On the contrary, it may increases. As seen from Table 1, the crystallinity and melting enthalpy of grafted PP fibers increased slightly compared to virginal PP.

\subsection{Effects of monomer concentration on grafting level of $P P$}

Figure 6 showed the effect of monomer (BA and LA) concentration on the grafting percentage. It can be seen that the grafting percentage increases initially with the concentration of the monomers when reaches a top value, and then decreases slightly. BA reached its top grafting level of $29.90 \%$ at the concentration of $7.5 \%$ while LA reached its top grafting level of $20.53 \%$ at the concentration of $10 \%$. Grafting level is highly related to scavenging ability for the free radicals and diffusivity to PP substrate of monomers. It is easy to understand that more monomers will be impregnated into the matrix as the concentration of monomer is high. However, the amount of homopolymers increases with the monomer concentration, and the homopolymers have been removed in the extraction process. It can be deduced that all the active sites on the PP substrate can be grafted when the concentration of the monomer is high enough. The initial increase in the grafting level is caused by the increased availability of free radicals for the chain transfer to polymer backbone. But when the concentration of the monomer exceeds a value, the average molecular weight of the side chains is reduced as well as the diffusivity of monomers, and the degree of homopolymerization and viscosity of solutions are increased. Both of these result in the reduction of the grafting level.

\subsection{Effects of cross linking reagent concentration on grafting level of $P P$}

The influence of divinylbenzene (DVB) on the grafting degree is shown in Figure 7. The grafting degree increased initially and dramatically with the presence of DVB, reached a top value and then decreased. At the concentration of $2 \%$, grafting levels of BA and LA reached a peak value of $20.53 \%$ and $21.64 \%$, respectively. Cross linking reagent with two double bonds has higher activity compared to acrylate. With a low DVB concentration, the grafting chains to PP films may be mostly cross linking reagent but not acrylate, which leads to the reduction of grafting level; With a high enough DVB concentration, the degree of homopolymerization of monomer acrylates are dramatically increased, which 
inhibited the diffusion of monomers to free radicals and thus interfered with the process of grafting reaction.

\section{Conclusions}

(1) Virginal and grafted PP fibers was investigated by IR, TG and DSC, and SEM, which showed that both LA and BA have been grafted onto PP.

(2) The maximum degree of grafting BA was $20.53 \%$, grafting percentage increased initially with the concentration of the monomer and cross linking reagent when reaching a top value, and then decreased, which indicated that excess higher or lower concentration of the monomer and cross linking reagent could lead to the reduction of grafting levels. The maximum degree of grafting LA was $29.90 \%$, its grafting percentage was similar to BA, which indicated that whether excess higher or lower concentration of he monomer and cross linking reagent was unfavorable to grafting reaction.

\section{References}

Bhuvanesh, G., Rachna, J., Nishat, A., et al.(2006). Radiat. Phys. Chem, 75: 161 167.

Chen, J., \& Lu, Y.C. (1999). Journal of Radiation Research and Radiation Processing, 17(4):193 198.

Chen, X.T., Tang, X.D., \& Zhang, M.Z. (2005). Ion Exchange and Adsorption, 21(6): 536 54.

Cho, J.D., Kim, S.G., \& Hong, J.W. (2006) J. Appl. Poly. Sci., 99: 1446 1461.

Dong, Y., Lan, X.Z., \& Li, J.X. (2006). Applied Chemical Industry, (8) :569 572.

Huang, Z.Z., Lin, Z.D., \& Cai, Z.W., et al. (2004). Preparation, structure and properties of polypropylene grafted with acrylic acid. Engineering Plastics Application, 32(2):71 74.

Park, H.J., \& Na, C.K. (2006) J. Colloid. Interface. Sci., 301: 46 54.

Ying, J.H., \& Mo, Z.S. (2001). Modern Polymer Physics. Beijing: Science press.

Zhang, Y.D., \& Chen, D.T. (1999). Polymer Materials Science \& Engineering, 15(5):79 82.

Zhu, C.S. (2004). Structure Analysis of Polymers. Beijing: Science press.

Vahdata, A., Bahramia. H., \& Ansaria. N., et al. (2007). Radiat. Phys. Chem., 76: 787 793.

Table 1. Related DSC parameters of virginal PP, PP-g-LA and PP-g-BA.

\begin{tabular}{|c|c|c|c|c|}
\hline Samples and Grafting degree (\%) & $\mathrm{T}_{\mathrm{m}}\left({ }^{\circ} \mathrm{C}\right)$ & $\triangle \mathrm{H}_{\mathrm{f}(\mathrm{fiber})}(\mathrm{J} / \mathrm{g})$ & $\triangle \mathrm{H}_{\mathrm{f}(\mathrm{PP})}(\mathrm{J} / \mathrm{g})$ & $\mathrm{W}_{\mathrm{c}}(\%)$ \\
\hline $1 \mathrm{PP}$ & 163.8 & 88.36 & 88.36 & 42.28 \\
\hline 2 PP-g-LA (15.75\%) & 162.7 & 79.27 & 94.08 & 45.01 \\
\hline 3 PP-g-BA (15.88\%) & 163.4 & 77.79 & 90.14 & 43.13 \\
\hline
\end{tabular}




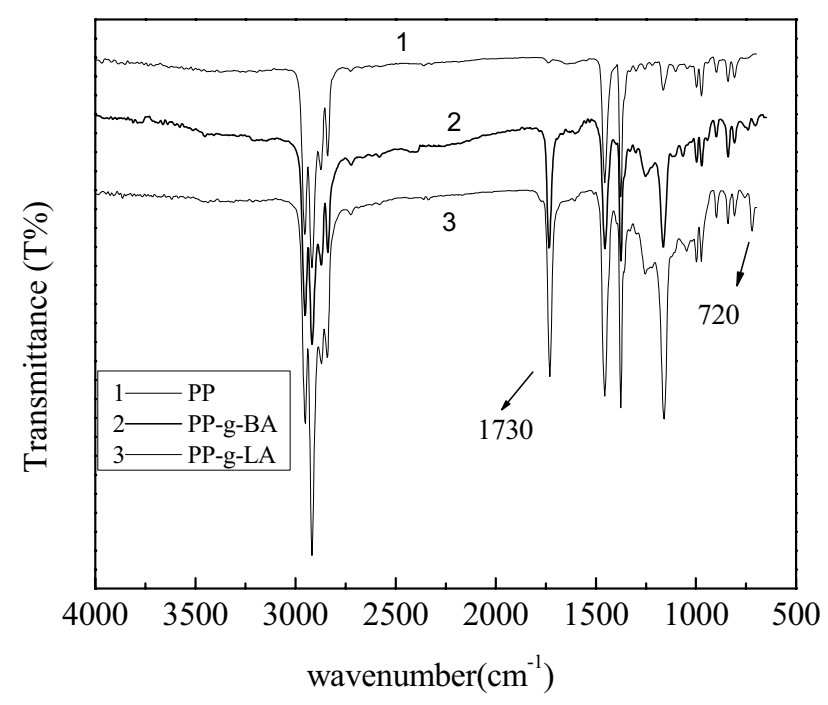

Figure 1. IR spectra of virginal PP (a), PP-g-LA (b), and PP-g-BA (c).

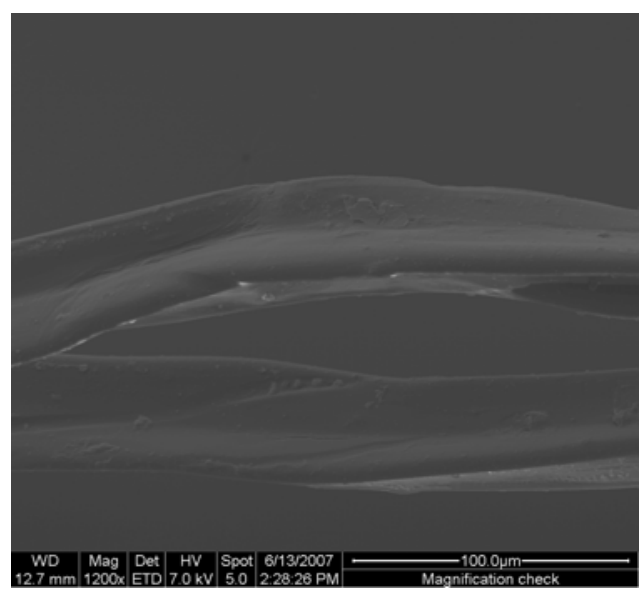

(a) PP

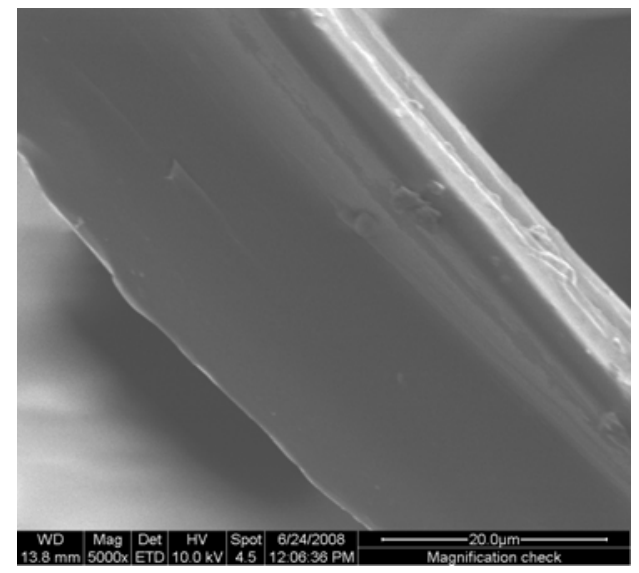

(c) PP

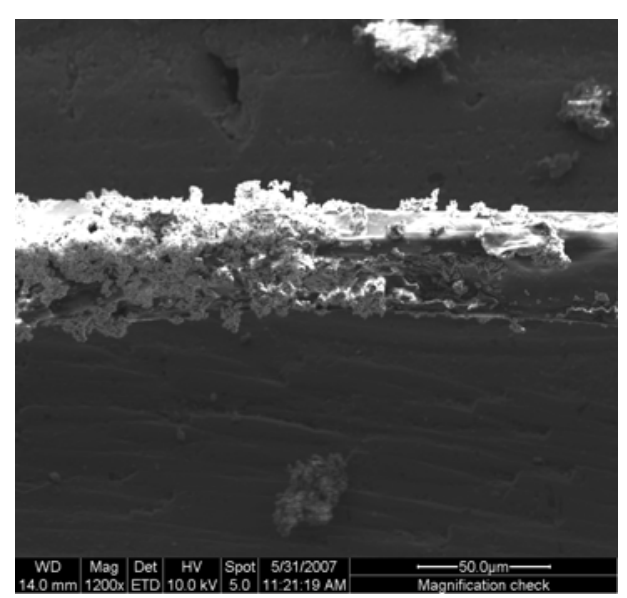

(b) PP-g-LA

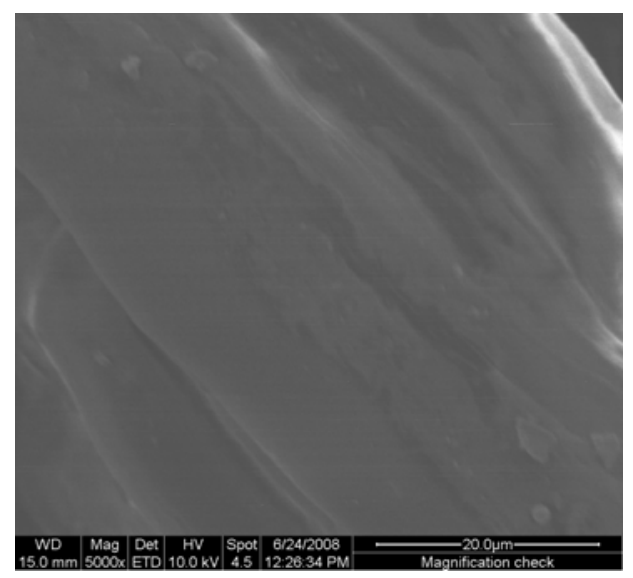

(d) PP-g-BA

Figure 2. SEM images of virginal PP (a), PP-g-LA (b), and PP-g-BA (c) 


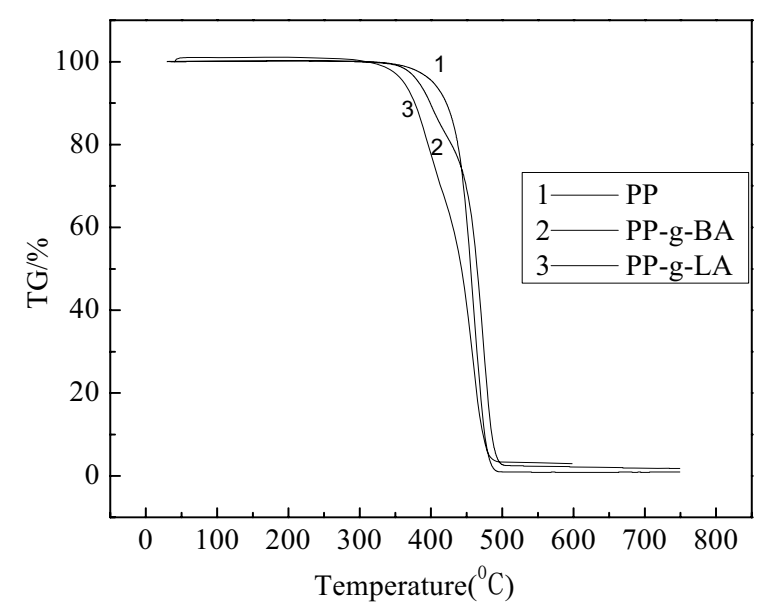

Figure 3. TG analysis of virginal PP (a), PP-g-LA (b), and PP-g-BA (c)

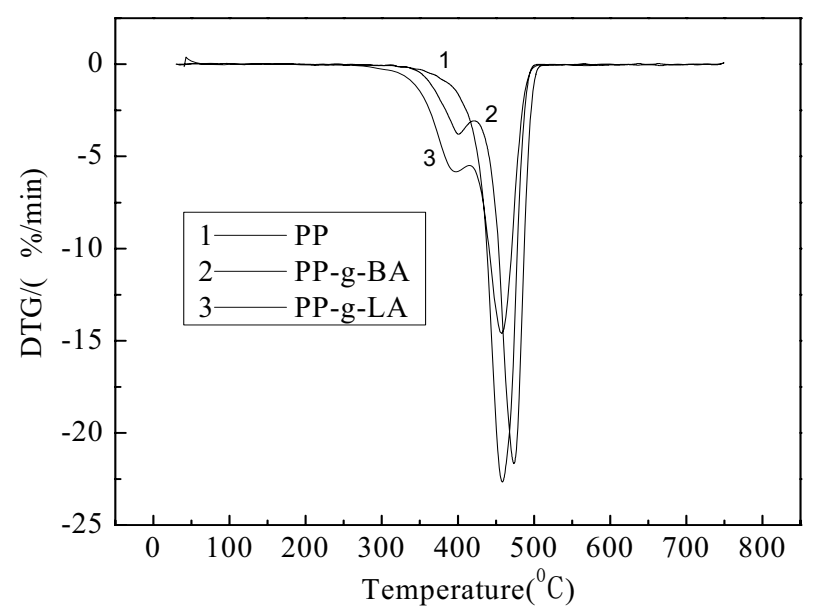

Figure 4. DTG analysis of virginal PP (a), PP-g-LA (b), and PP-g-BA (c)

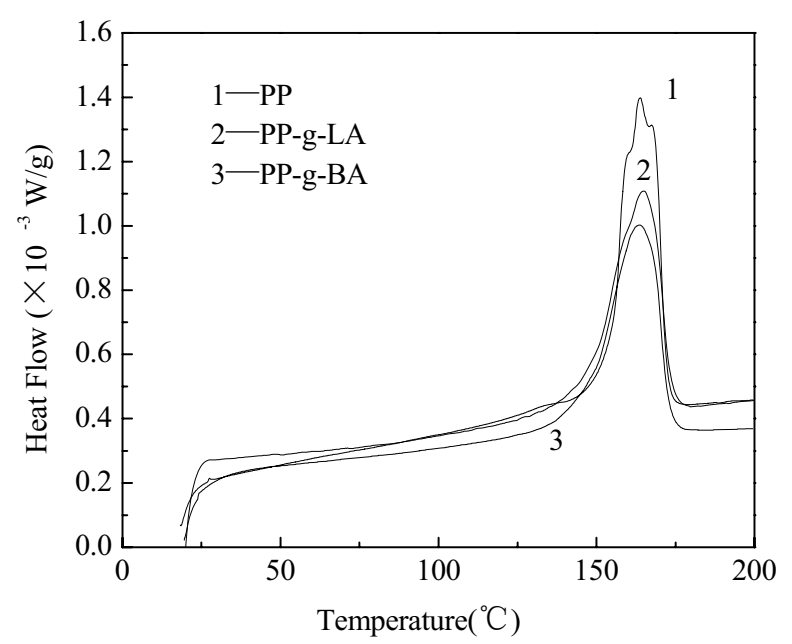

Figure 5. DSC thermographs of virginal PP (a), PP-g-LA (b), and PP-g-BA (c) 


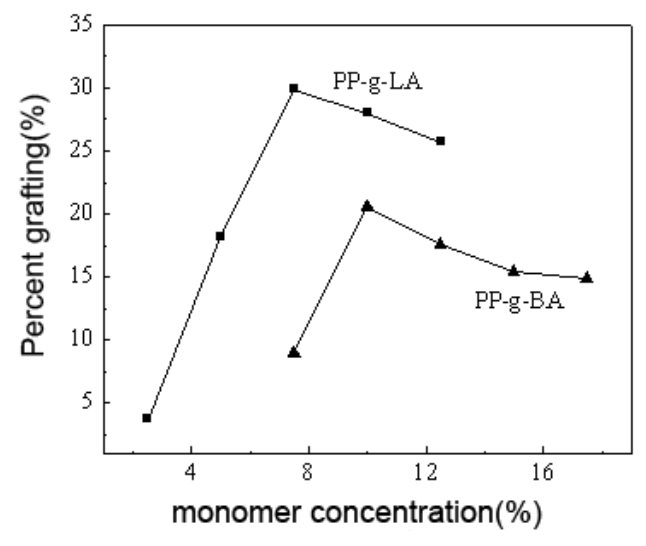

Figure 6. Relation between monomer concentration and grafting level

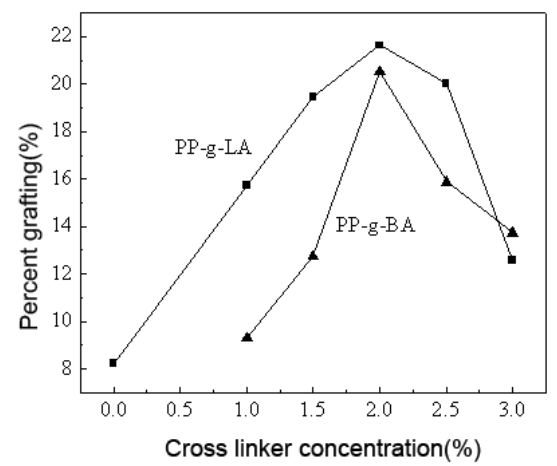

Figure 7. Relation between cross linking reagent concentration and grafting level 